\title{
The sky pattern of the linearized gravitational memory effect
}

\author{
Thomas Mädler ${ }^{1} \ddagger$ and Jeffrey Winicour ${ }^{2,3}$ \\ ${ }^{1}$ Institute of Astronomy, University of Cambridge, Madingley Road, Cambridge, \\ CB3 0HA, UK \\ ${ }^{2}$ Department of Physics and Astronomy \\ University of Pittsburgh, Pittsburgh, PA 15260, USA \\ ${ }^{3}$ Max-Planck-Institut für Gravitationsphysik, Albert-Einstein-Institut, \\ 14476 Golm, Germany
}

\begin{abstract}
The gravitational memory effect leads to a net displacement in the relative positions of test particles. This memory is related to the change in the strain of the gravitational radiation field between infinite past and infinite future retarded times. There are three known sources of the memory effect: (i) the loss of energy to future null infinity by massless fields or particles, (ii) the ejection of massive particles to infinity from a bound system and (iii) homogeneous, source-free gravitational waves. In the context of linearized theory, we show that asymptotic conditions controlling these known sources of the gravitational memory effect rule out any other possible sources with physically reasonable stress-energy tensors. Except for the source-free gravitational waves, the two other known sources produce gravitational memory with E-mode radiation strain, characterized by a certain curl-free sky pattern of their polarization. Thus our results show that the only known source of B-mode gravitational memory is of primordial origin, corresponding in the linearized theory to a homogeneous wave entering from past null infinity.

PACS numbers: $\quad$ 04.20.-q, 04.20.Cv, 04.20.Ex, 04.25.D-, 04.30-w
\end{abstract}

\section{Introduction}

The gravitational memory effect produces a net displacement between test particles after the passage of a gravitational wave. The effect was initially recognized in linearized gravity where the radiation memory is produced by an exploding system of massive particles which escape to infinity [1]. Independently such burst memory was found as a zero frequency mode of gravitational radiation in studies of the collision of relativistic point particles [2] and in studies of astrophysical gravitational wave emission [3]. A nonlinear form of radiation memory was discovered by Christodoulou [4], which was then related [5, 6] to the flow of gravitons to null infinity. Subsequent studies showed that even in linearized gravitational theory the analogous energy transport to future null

$\ddagger$ Email:tm513@cam.ac.uk 
infinity $\mathcal{I}^{+}$by massless particles or fields (neutrinos, electromagnetic radiation, etc.) also produce gravitational radiation memory [7, 8, 9]; so that rather than nonlinear memory this is now referred to as null memory. Despite the stunning recent observation of gravitational waves by the LIGO-Virgo consortium [10], the detection of gravitational memory is more demanding due to the current insensitivity of the LIGO detectors, as well as pulsar timing arrays [11], to the long rise-time of typical memory signals [12].

An overlooked aspect of gravitational radiation memory has been its global sky pattern, which can be decomposed into $\mathrm{E}$ and $\mathrm{B}$ modes similar to the decomposition of electromagnetic radiation, as explained in Sec. 2. Recently, it has been shown that both the gravitational burst memory due to ejected particles and the null memory are purely E-mode [13], where it was also pointed out that B-mode gravitational memory could be produced by a homogeneous, source-free gravitational wave, as we explicitly demonstrate in Sec. 5.2.2.

In this paper, we refine and elaborate the global properties of gravitational radiation memory in the context of linearized gravitational theory. We show that if burst memory (or its time reversed counterpart consisting of the capture of particles incident from infinity) and homogeneous wave memory are eliminated by a weak asymptotic stationarity condition then null memory is the only possible form of gravitational memory arising from matter with a physically reasonable stress-energy tensor. Except for homogeneous wave memory, the other two known sources (burst and null) produce E-mode gravitational memory. Thus our results imply that B-mode memory is of primordial origin, corresponding in the linearized theory to a homogeneous wave entering from past null infinity. These results apply to non-compact matter sources with isotropic stress, scalar fields and electromagnetic fields.

The gravitational memory effect is an asymptotic feature which arises from the difference in the radiation strain at $\mathcal{I}^{+}$between infinite past and infinite future retarded times. As we discuss in Sec. 2, there is a connection between the gravitational memory effect and the supertranslation freedom in the Bondi-Metzner-Sachs (BMS) asymptotic symmetry group [14]. For this reason, we base our work upon a linearized version of the null hypersurface formulation of the Einstein equations pioneered by Bondi et al [15] and by Sachs [16]. The aspects of the Bondi-Sachs formulation related to gravitational wave memory are reviewed in Sec. 3. While this is a metric based approach, we concentrate on gauge invariant quantities. For an alternative covariant formalism in terms of the Weyl tensor see [17].

In Sec. 4, we present the underlying assumptions regarding asymptotic flatness and the asymptotic conditions on the matter stress-energy tensor. In Sec. 5, we discuss a weak asymptotic stationarity condition which controls incoming radiation fields and rules out two of the known sources of the gravitational memory effect: the burst memory and the homogeneous wave memory. The weak asymptotic stationarity condition requires that the leading $r^{-5}$ coefficient of the Newman-Penrose curvature component $\Psi_{0}$ be stationary in the retarded time limits $u= \pm \infty$. Our main results for E-mode memory are given in Sec. 6.1, and those for B-mode memory, in Sec. 6.2, The role of 
matter fields is discussed in Sec. 6.3.

Our approach is based upon a previous study of the global properties of electromagnetic radiation memory [13], which showed that only the known current distributions which carry charge to infinity can produce E-mode memory [18] and that B-mode electromagnetic radiation memory cannot be be produced by a physically realistic charge-current distribution. In the course of this work, it was realized that the version of asymptotic stationarity condition assumed in [13] was unnecessarily strong. In Appendix A, we revisit the electromagnetic memory results under a weaker condition of asymptotic stationarity analogous to the gravitational version presented here.

As pointed out in [18], the electromagnetic memory effect is more dramatic than the gravitational effect. Electromagnetic waves produced by the ejection of a charged particle give rise to a net momentum kick to test charges in the radiation zone, as opposed to the displacement of test masses in the gravitational effect. Other recent work on aspects of the memory effect addresses its role in cosmological effects [19, 20, 21, 22], in binary neutron star mergers [23], in higher dimensional theories [24], in properties of angular momentum [25, 26], in the supertranslation freedom [27] and in the black hole information paradox [28, 45].

We use geometric units in which the Einsteinian gravitational constant is $\kappa=8 \pi$. The signature of the metric is +2 and we use the conventions of [31] for the curvature. For reference, a list of the linearized Christoffel symbols is provided in Appendix B

\section{Radiation fields on the celestial sphere, geometrical framework}

In linearized theory, gravitational radiation is described by a trace free strain tensor on the celestial sphere $x^{A}=(\theta, \phi)$,

$$
\sigma_{A B}\left(u, x^{A}\right), \quad q^{A B} \sigma_{A B}=0,
$$

where $q_{A B} d x^{A} d x^{B}=d \theta^{2}+\sin ^{2} \theta d \phi^{2}$ is the standard unit sphere metric and $u$ is the retarded time. The radiation memory is determined by the change in the strain between infinite future and past retarded time,

$$
\Delta \sigma_{A B}:=\sigma_{A B}(u=\infty, \theta, \phi)-\sigma_{A B}(u=-\infty, \theta, \phi) .
$$

This produces a net displacement in the relative angular position of distant test particles

$$
\Delta\left(x_{2}^{A}-x_{1}^{A}\right)=q^{A B} \Delta \sigma_{B C}\left(x_{2}^{C}-x_{1}^{C}\right),
$$

where we use the notation $\Delta F\left(x^{C}\right):=\left.F\left(u, x^{C}\right)\right|_{u=\infty}-\left.F\left(u, x^{C}\right)\right|_{u=-\infty}$.

Similarly, the electromagnetic radiation pattern at future null infinity $\mathcal{I}^{+}$is characterised by the angular components of the (rescaled) electric field $E_{A}\left(u, x^{B}\right)$. The $\mathrm{E}$ and $\mathrm{B}$ mode classification results from decomposing the electric field in terms of a gradient and the dual of a gradient,

$$
E_{A}=D_{A} \Phi_{[e]}+\epsilon_{C A} D^{C} \Phi_{[b]},
$$


where $D_{A}$ is the covariant derivative and $\epsilon_{A B}$ is the antisymmetric surface area tensor on the unit sphere, respectively. The real scalar fields $\Phi_{[e]}$ and $\Phi_{[b]}$ correspond to the E-mode and B-mode, respectively.

A compact way to describe this decomposition is in terms of a complex polarisation vector $q_{A}$ satisfying

$$
q_{A B}=q_{(A} \bar{q}_{B)}, \quad \epsilon_{A B}=i q_{[A} \bar{q}_{B]}, \quad q^{A} \bar{q}_{A}=2, \quad q^{A} q_{A}=0,
$$

e.g. $q_{A}=(1, i \sin \theta)$ for the standard form of the unit sphere metric and where $(A B)$ and $[A B]$ denote the standard notation for symmetrization and antisymmetrization of indices. Then the electromagnetic radiation is represented by the spin-weight 1 field

$$
q^{A} E_{A}=q^{A}\left(D_{A} \Phi_{[e]}+\epsilon_{A}^{C} D_{C} \Phi_{[b]}\right)=ð\left(\Phi_{[e]}+i \Phi_{[b]}\right),
$$

where $\partial$ is the Newman-Penrose spin-weight raising operator [29, 37].

The analogous decomposition of the gravitational radiation field can be made by first noting that the strain can be represented in terms of a displacement vector field $\xi^{A}$ by

$$
\sigma_{A B}=\frac{1}{2}\left(D_{A} \xi_{B}+D_{B} \xi_{A}\right)-\frac{1}{4} q_{A B} D^{C} \xi_{C} .
$$

Given two real scalar fields $\Sigma_{[e]}$ and $\Sigma_{[b]}$ that form the complex scalar field $\Sigma:=$ $\Sigma_{[e]}+i \Sigma_{[b]}$, the decomposition

$$
\xi_{A}=D_{A} \Sigma_{[e]}+\epsilon_{B A} D^{B} \Sigma_{[b]}
$$

leads to the spin-weight 2 representation of the strain,

$$
\sigma:=q^{A} q^{B} \sigma_{A B}=q^{A} q^{B} D_{A} D_{B}\left(\Sigma_{[e]}+i \Sigma_{[b]}\right)=\partial^{2}\left(\Sigma_{[e]}+i \Sigma_{[b]}\right)=ð^{2} \Sigma .
$$

In spin-weight terminology, $\Sigma_{[e]}$ and $\Sigma_{[b]}$ represent the "electric" and "magnetic" parts of the strain, corresponding to the $\mathrm{E}$ and $\mathrm{B}$ radiation modes. Here $\Sigma$ is the spin-weight

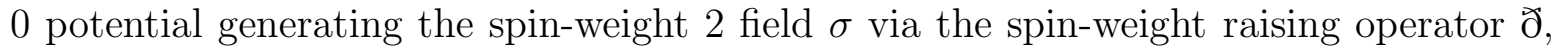
according to (9). As we will make extensive use of the spin-weight calculus, if $J_{A_{1} \ldots A_{n}}$ is a symmetric trace free tensor field on the sphere and $\check{\partial}^{n} J=q^{A_{1}} \ldots q^{A_{n}} J_{A_{1} \ldots A_{n}}$, we denote the real part of the corresponding spin-weight 0 potential $J$ by $J_{[e]}$ and its imaginary part by $J_{[b]}$.

In the electromagnetic case, the $\vec{E}$ and $\vec{B}$ radiation fields have equal magnitude and are orthogonal in direction. Thus the electromagnetic radiation pattern and its memory could be equally well described in terms its $\vec{B}$ field. Similarly, in the gravitational case, the radiation fields corresponding to $\vec{E}$ and $\vec{B}$, in an inertial frame picked out by a time-like vector $T^{a}$, arise from the components of the Weyl tensor and its dual,

$$
\mathbf{E}=T^{a} T^{b} q^{A} q^{B} C_{a A b B}, \quad \mathbf{B}=-\frac{1}{2} T^{a} T^{b} q^{A} q^{B} \epsilon_{a A c C} C_{b B}^{c C},
$$

where $\epsilon_{0123}=1$ is the four dimensional Levi Civita tensor. Here it follows from the Petrov type $N$ of the radiation field that the spin-weight 2 fields $\mathbf{E}$ and $\mathbf{B}$ are equal in magnitude and oriented at $45^{\circ}$, i.e. $\mathbf{E} \rightarrow \mathbf{B}$ under the spin rotation $q^{A} \rightarrow e^{i \pi / 4} q^{A}$. The 
leading coefficients of the electric and magnetic parts of the Weyl tensor in an expansion at $\mathcal{I}^{+}$are related to the strain according to

$$
E\left(u, x^{A}\right)=\partial_{u}^{2} \sigma\left(u, x^{A}\right), \quad B\left(u, x^{A}\right)=i \partial_{u}^{2} \sigma\left(u, x^{A}\right) .
$$

For our purposes, it will be useful to deal with the strain but the Weyl tensor representation has the advantage of being gauge invariant. The strain has gauge freedom

$$
\sigma \rightarrow \sigma+\check{\partial}^{2} \alpha
$$

under a retarded time transformation $u \rightarrow u+\alpha\left(x^{A}\right)$, which corresponds to the supertranslation freedom in the Bondi-Metzner-Sachs asymptotic symmetry group [14, 29]. Thus, on comparison with (12), the E-mode component of the strain can be gauged away during any stationary epoch. However, the E-mode of the memory (2) is gauge invariant and can be considered to be a supertranslation shift between the two preferred gauges for the strain picked out at $u= \pm \infty$. It is curious, and perhaps of some deeper significance, that in the electromagnetic case the E-mode radiation memory is also related to a gauge shift, in that case with respect to the vector potential [13] (see Appendix A).

\section{Linearized Bondi-Sachs Framework}

It will be useful to refer to three separate coordinate systems. A Cartesian inertial system $\left(t, x^{i}\right)=(t, x, y, z)$, the associated spherical coordinate system $\left(t, r, x^{A}\right)=$ $(t, r, \theta, \phi), r^{2}=x^{2}+y^{2}+z^{2}$, and the associated outgoing null spherical coordinate system $x^{\alpha}=\left(u, r, x^{A}\right)$, with retarded time $u=t-r$ and vertices of the null cones at $r=0$. In these retarded null coordinates, the Bondi-Sachs line element is

$$
d s^{2}=g_{u u} d u^{2}+2 g_{u r} d u d r+2 g_{u A} d u d x^{A}+r^{2} h_{A B} d x^{A} d x^{B},
$$

where the use of an areal radial coordinate $r$ implies that $\operatorname{det}\left(h_{A B}\right)=\operatorname{det}\left(q_{A B}\right)$. In the linearized approximation off the Minkowski background metric $\eta_{a b}$,

$$
\begin{aligned}
& g_{u u} \approx-1-2 \beta-W, \\
& g_{u r} \approx-1-2 \beta, \\
& g_{u A} \approx-r^{2} q_{A B} U^{B}, \\
& h_{A B} \approx q_{A B}+J_{A B}, \quad q^{A B} J_{A B}=0 .
\end{aligned}
$$

In the following we treat $\left(\beta, W, U^{A}, J_{A B}\right)$ as linearized quantities and neglect higher order terms. In this approximation, the non-zero contravariant metric components are

$$
\begin{aligned}
& g^{u r}=-1+2 \beta, \\
& g^{r r}=1-2 \beta+W, \\
& g^{r A}=-U^{A}, \\
& g^{A B}=r^{-2}\left(q^{A B}-J^{A B}\right), \quad J^{A B}:=q^{A C} q^{B D} J_{C D} .
\end{aligned}
$$

Instead of working with the metric variables, we introduce spin-weighted fields and express covariant derivative $D_{A}$ with respect to the unit sphere metric in terms of the 
ठे operator [29, 32]. For example, we represent the traceless symmetric tensor $J_{A B}$ by the pure spin-weight 2 field $\mathcal{J}=q^{A} q^{B} J_{A B}$ and write

$$
\text { ð } \mathcal{J}=q^{A} q^{B} q^{C} D_{C} J_{A B}, \quad \overline{\bar{\gamma}} \mathcal{J}=q^{A} q^{B} \bar{q}^{C} D_{C} J_{A B} .
$$

A spin-weight $s$ field $\mathcal{F}\left(x^{a}\right)$ satisfies the commutation relation $[\overline{\widetilde{\jmath}}, \widetilde{\partial}] \mathcal{F}=2 s \mathcal{F}$.

The linearized metric is determined by $\beta, W$, the spin-weight 1 field $\mathcal{U}=q_{A} U^{A}$ and the spin-weight 2 field $\mathcal{J}=q^{A} q^{B} J_{A B}$. In the same way, we represent the components $\rho_{a b}$ of the reduced stress-energy tensor of the standard matter stress-energy tensor $T_{a b}$,

$$
\rho_{a b}:=T_{a b}-\frac{1}{2} \eta_{a b} T_{c}^{c}
$$

by the spin-weighted fields

$$
\mathcal{N}=q^{A} \rho_{u A}, \quad \mathcal{P}=q^{A} \rho_{r A}, \quad \mathcal{S}=q^{A} q^{B} \rho_{A B},
$$

and the spin weight 0 field $S_{0}=q^{A} \bar{q}^{B} \rho_{A B}$.

A spin-weight $s$ field $\mathcal{F}\left(x^{a}\right)$ can be expanded in terms of spin-weighted harmonics ${ }_{s} Y_{l m}\left(x^{A}\right)(l \geq s)$

$$
\mathcal{F}\left(x^{a}\right)=\sum_{l=s}^{\infty} \sum_{m=-l}^{l} f_{l m}(u, r){ }_{s} Y_{l m}\left(x^{A}\right) .
$$

Here the ${ }_{s} Y_{l m}$ are generated by applying $\partial^{s}$ to the standard spherical harmonics $Y_{l m}\left(x^{A}\right)$. §. By this procedure, we can introduce a complex spin-weight 0 potential $F$ such that $\mathcal{F}=\partial^{s} F$. Hereafter, we denote any spin-weight $s \neq 0$ quantity by a scripted font, e.g. $\mathcal{F}$, and the its spin-weight 0 potential with the corresponding Roman font, e.g $F$. This procedure is not a priori unique since $\partial^{s} Y_{l m}=0$ for $l<s$. We remove this ambiguity by imposing the convention that for a spin-weight s field $\mathcal{F}$ its corresponding potential $F$ contains no harmonics with $l<s$.

Application of this notation to the metric and matter variables leads to the spinweight 0 potentials

$$
\mathcal{U}=ð U, \quad \mathcal{J}=\mathrm{\partial}^{2} J, \quad \mathcal{N}=ð N, \quad \mathcal{P}=ð P, \quad \mathcal{S}=\mathrm{\partial}^{2} S .
$$

Note that due to the above convention, $U, P$ and $N$ have no $l=0$ modes (which otherwise would not be of physical significance). Similarly, $J$ and $S$ have no $l=0$ and no $l=1$ modes. This notation leads to a natural decomposition of the linearized field equations into their E and B-modes corresponding, respectively, to their real and imaginary parts, as in the decomposition of the strain (9).

\subsection{Einstein Equations}

We express the linearized Einstein equations in the form

$$
R_{a b}=\kappa \rho_{a b} .
$$

$\S$ The $s<0$ case is obtained by exchanging $ð$ by $\bar{\partial}$. 
Following the formalism in [33, 34], they decompose into the hypersurface equations $\partial_{r} \beta=\frac{\kappa}{4} r \rho_{r r}$

$\partial_{r}\left(r^{4} \partial_{r} \mathcal{U}\right)=2 r^{4} \partial_{r}\left(\frac{1}{r^{2}} \precsim \beta\right)-\frac{1}{2} r^{2} \bar{\partial} \partial_{r} \mathcal{J}+2 \kappa r^{2} \mathcal{P}$,

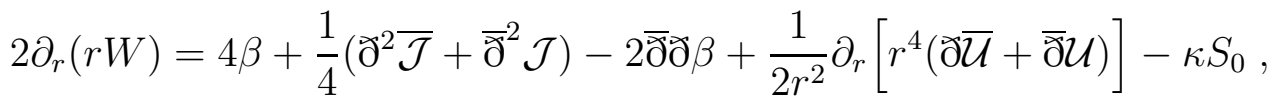

the complex evolution equation

$r \partial_{r} \partial_{u}(r \mathcal{J})=\frac{1}{2} \partial_{r}\left(r^{2} \partial_{r} \mathcal{J}\right)-ð \partial_{r}\left(r^{2} \mathcal{U}\right)+2 ð^{2} \beta+\kappa \mathcal{S}$,

and the supplementary conditions

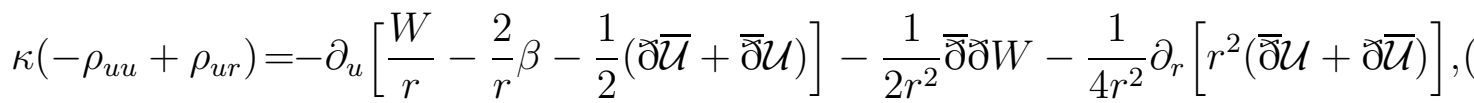

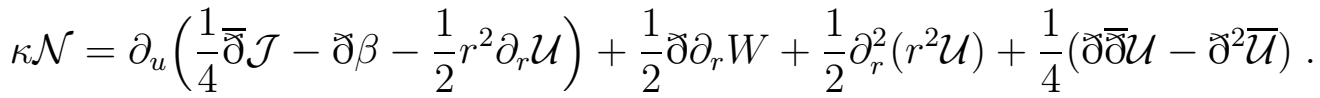

The supplementary conditions can be interpreted as conservation equations [35, 36]. It follows from the Bianchi identities and the matter conservation laws that if the hypersurface and evolution equations are satisfied then the supplementary conditions are automatically satisfied if they are satisfied on a worldtube $r=R\left(u, x^{A}\right.$ ) (or if the vertex world line $r=0$ is nonsingular).

Rewritten in terms of the spin-weight 0 fields $(U, J, N, P, S)$, these linearized equations reduce to the hypersurface equations

$\partial_{r} \beta=\frac{\kappa}{4} r \rho_{r r}$

$\partial_{r}\left(r^{4} \partial_{r} U\right)=2 r^{4} \partial_{r}\left(\frac{1}{r^{2}} \beta\right)-\frac{1}{2}(\bar{\varpi} \check{\partial}+2) r^{2} \partial_{r} J+2 \kappa r^{2} P$,

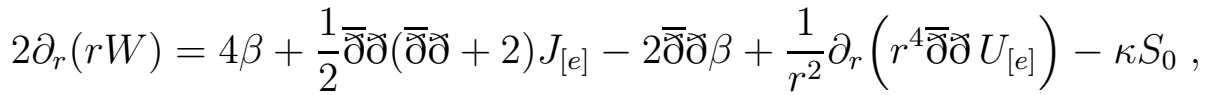

the evolution equation

$r \partial_{r} \partial_{u}(r J)=\frac{1}{2} \partial_{r}\left(r^{2} \partial_{r} J\right)-\partial_{r}\left(r^{2} U\right)+2 \beta+\kappa S$,

and the supplementary equations

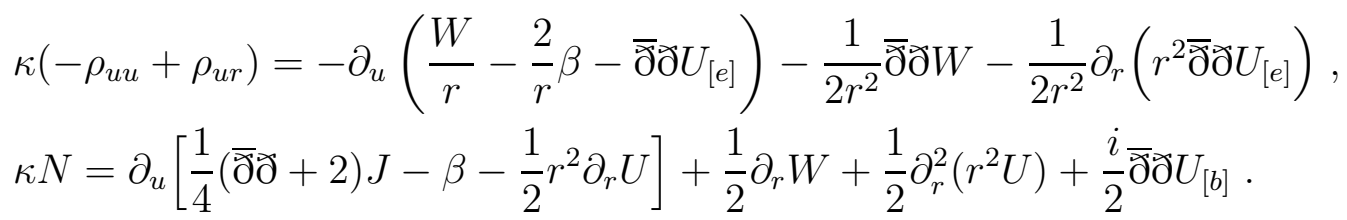

Given the gravitational data $J$ on an initial null hypersurface $u_{0}$ and the matter data $\rho_{r r}, P, S$ and $S_{0}$, the hypersurface and evolution equations consist of radial differential equations which can be integrated at retarded time $u_{0}$ to determine $\beta, U, W, \partial_{u} J$, in that sequential order. The corresponding integration constants are a mixture of physical properties of the system, e.g. the mass and angular momentum aspects, and gauge information. As explained in Sec. 4.2, it is possible to use the gauge freedom to set

$$
\left.\beta\left(u, r, x^{A}\right)\right|_{r=\infty}=\left.U\left(u, r, x^{A}\right)\right|_{r=\infty}=\left.J\left(u, r, x^{A}\right)\right|_{r=\infty}=0
$$


The Newman-Penrose Weyl component $\Psi_{0}$ [39, 40] is given in terms of the gravitational data by

$$
\Psi_{0}=-\frac{1}{4} C_{a b c d} K^{a} Q^{b} K^{c} Q^{d}=\frac{1}{8 r^{2}} \partial_{r}\left(r^{2} \partial_{r} ð^{2} J\right),
$$

where $C_{a b c d}$ is the Weyl tensor. Here $Q_{a}=\left(0,0, r q_{A}\right)$ and

$$
K_{a}=-\nabla_{a} u, \quad N_{a}=-\nabla_{a} v
$$

are the null vectors associated with the retarded time $u=t-r$ and the advanced time $v=t+r$, with normalization $K^{a} N_{a}=-2$.

\section{Asymptotic behavior}

We are interested in systems which are asymptotically flat at future null infinity [30]. In particular, we impose the peeling property which requires that the leading terms in the asymptotic expansion of the Bondi-Sachs metric can be expressed as a power series in $1 / r$. Thus, to the required power of $1 / r$, we assume that a field $F\left(x^{a}\right)$ describing the metric or matter has an expansion

$$
F\left(x^{a}\right)=F_{[0]}\left(u, x^{A}\right)+\frac{F_{[1]}\left(u, x^{A}\right)}{r}+\frac{F_{[2]}\left(u, x^{A}\right)}{r^{2}}+\frac{F_{[3]}\left(u, x^{A}\right)}{r^{4}}+\ldots .
$$

where the coefficients functions $F_{[n]}\left(u, x^{A}\right)$ are evaluated at $\mathcal{I}^{+}$. In particular, $F(u, r=$ $\left.\infty, x^{A}\right)=F_{[0]}\left(u, x^{A}\right)$.

We make the following physical assumptions on the matter at null infinity:

(i) The total energy, momentum and angular momentum of the matter, and their related fluxes are finite.

(ii) The dominant energy condition holds.

(iii) The matter stress-energy tensor satisfies the local conservation law $\nabla_{a} T_{b}^{a}=0$.

These matter conditions are complemented by asymptotic flatness and gauge conditions on the perturbed metric.

\subsection{Matter asymptotics}

The most general asymptotic conditions on a stress-energy tensor $T_{a b}$ are that the total energy, momentum and angular momentum, and their fluxes are finite. These conditions indirectly apply to the stresses by requiring a dominant energy condition.

The condition that the total energy and momentum content of the matter be finite, i.e. $\int_{0}^{\infty} \xi^{a} T_{a}{ }^{u} r^{2} d r$ converges for every background translational Killing vector $\xi^{a}$, implies

$$
T_{u}^{u}=O\left(r^{-4}\right), T_{r}^{u}=O\left(r^{-4}\right), T_{A}^{u}=O\left(r^{-3}\right),
$$

Index symmetry of $T_{a b}$ then implies

$$
T_{r}^{r}=O\left(r^{-4}\right), T_{r}^{A}=O\left(r^{-5}\right) .
$$

\| The factor $1 / 4$ in (28) arises because of the normalization of the null vectors and $Q^{a}$. 
The condition that the matter transport finite energy-momentum and angular momentum to infinity, i.e. that the flux $\xi^{a} T_{a}{ }^{r} r^{2}$ be finite at $\mathcal{I}^{+}$for each Minkowski background space-time translational or rotational Killing vector $\xi^{a}$, requires

$$
T_{u}^{r}=O\left(r^{-2}\right), \quad T_{A}^{r}=O\left(r^{-2}\right), \quad T_{u}^{A}=O\left(r^{-4}\right),
$$

where we have again used index symmetry to constrain $T_{u}^{A}$. Furthermore, the requirement that the time integrated fluxes $\int_{-\infty}^{\infty} \xi^{a} T_{a}^{r} r^{2} d u$ also be finite gives stronger conditions in the infinite past and future (see also App. of [17]),

$$
T_{u}^{r}=O\left(r^{-3}\right), \quad T_{A}^{r}=O\left(r^{-3}\right), \quad T_{u}^{A}=O\left(r^{-5}\right), \quad u= \pm \infty
$$

The dominant energy condition requires that for any observer the velocity of the matter is alway smaller than the velocity of light. Specifically, for every unit timelike vector $\tau^{a}$, with $T_{a b} \tau^{a} \tau^{b} \geq 0$ and $T_{a b} \tau^{a}$ a non-spacelike vector, an equivalent formulation of the dominant energy condition is that in any orthonormal basis $\left(\tau^{a}, e_{(i)}^{a}\right), i=1,2,3$, $T_{a b} \tau^{a} \tau^{b} \geq\left|T_{(i)(j)}\right|$ for each $i, j$ [38]. This implies conditions on the stress components. The vector $\tau^{a}=\left(r^{-1 / 4}, \frac{1}{2} r^{1 / 4}, 0,0\right)$, with norm $\tau^{a} \tau_{a}=-\left(1+r^{-1 / 2}\right)$, approaches a unit timelike vector as $r \rightarrow \infty$. Together with (31d) this implies $T_{a b} \tau^{a} \tau^{b}=O\left(r^{-3.5}\right)$ at $u= \pm \infty$. Since we require that the leading terms in an asymptotic expansion be integer powers of $1 / r$, for an orthonormal base associated with $\tau^{a}$ at $\mathcal{I}^{+}$the dominant energy condition implies

$$
T_{B}^{A}=O\left(r^{-4}\right), \quad u= \pm \infty .
$$

The relations (31a)-(31e) manifest the most general asymptotic behaviour of the $T_{a b}$ under the given assumptions. Given these results, the leading terms in the conservation laws $\nabla_{b} T_{a}^{b}=0$ imply at $u= \pm \infty$

$$
0=\partial_{u} T_{u[4]}^{u}-T_{u[3]}^{r}=\partial_{u} T_{r[4]}^{u}=\partial_{u} T_{A[3]}^{u} .
$$

In terms of the reduced tensor $\rho_{a b}$, the asymptotic behaviour (31a)-(31e) correspond to

$$
\rho_{u u}=O\left(r^{-2}\right), \rho_{u r}=O\left(r^{-3}\right), \rho_{r r}=O\left(r^{-4}\right),
$$

and for the matter spin-weight 0 potentials

$$
N=O\left(r^{-2}\right), \quad P=O\left(r^{-3}\right) .
$$

Further, the finiteness of the time-integrated flux along with the dominant energy condition require at $u= \pm \infty$

$$
\rho_{u u}=O\left(r^{-3}\right), \quad N=O\left(r^{-3}\right), S_{0}=O\left(r^{-2}\right), \quad S=O\left(r^{-2}\right) .
$$

The conservations laws (32) in terms of $\rho_{a b}$ yield at $u= \pm \infty$

$$
0=\partial_{u} S_{0[2]}-2 \rho_{u u[3]}=\partial_{u} \rho_{r r[4]}=\partial_{u} P_{[3]} .
$$




\subsection{Spacetime asymptotics}

The asymptotic behavior of the matter tensor in the previous section ensures that the radial integration of the hypersurface and evolution equations is convergent as $r \rightarrow \infty$. Some of the integration constants involved in these radial integrals represent pure gauge effects and others represent physical quantities such as the mass and angular momentum aspects. Radiation memory is an asymptotic effect whose treatment is simplified by taking advantage of the asymptotic gauge freedom.

For a given gauge transformation $\xi^{a}$ (i.e. a linearized diffeomorphism), the linearized metric undergoes the gauge transformation [31]

$$
\delta_{\underline{\xi}} g^{a b}=g^{a c} \partial_{c} \xi^{b}+g^{c b} \partial_{c} \xi^{a}-\xi^{c} \partial_{c} g^{a b} .
$$

This gauge freedom is subject to the Bondi-Sachs coordinate conditions $\delta_{\xi} g^{u u}=0$, $\delta_{\underline{\xi}} g^{u A}=0$ and $g_{A B} \delta_{\underline{\xi}} g^{A B}=0$ [14, 15], which leads to the functional dependence

$$
\xi^{u}=\alpha\left(u, x^{B}\right), \quad \xi^{A}=f^{A}\left(u, x^{B}\right)-\frac{1}{r} D^{A} \alpha, \quad \xi^{r}=-\frac{r}{2} D_{B} \xi^{B} .
$$

By setting $q_{A} \xi^{A}=\xi$ and $q_{A} f^{A}=ð f$, in terms of a complex scalar field $f\left(u, x^{A}\right)$, we obtain

$$
\xi=ð f-r^{-1} \partial \alpha, \quad \xi^{r}=-\frac{r}{2} \bar{ð} \precsim f_{[e]}+\frac{1}{2} \bar{\varnothing} ð \alpha .
$$

This gives rise to the following gauge freedom in the linearized metric variables:

$$
\begin{aligned}
& 2 \delta_{\underline{\xi}} \beta=-\partial_{u} \alpha-\partial_{r} \xi^{r}=-\partial_{u} \alpha+\frac{1}{2} ð \bar{\gamma} f_{[e]}, \\
& \delta_{\underline{\xi}} \mathcal{U}=\left(\partial_{u}-\partial_{r}\right) \xi-\frac{1}{r^{2}} ð \xi^{r}=\partial_{u} \partial f+\frac{1}{2 r} ð\left(\bar{ð} \precsim f_{[e]}-2 \partial_{u} \alpha\right)+\frac{1}{2 r^{2}} ð(\bar{\partial} ð+2) \alpha, \\
& \delta_{\underline{\xi}} \mathcal{J}=-\check{\partial}^{2} f+\frac{1}{r} ð^{2} \alpha,
\end{aligned}
$$

and

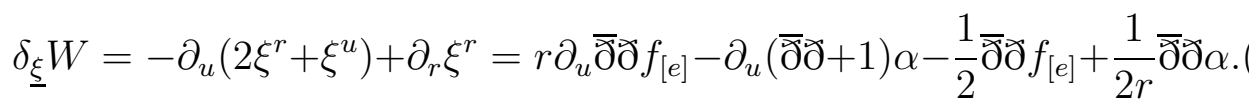

This gauge freedom allows us to fix the integration constants at $\mathcal{I}^{+}$. In particular,

$$
\lim _{r \rightarrow \infty} \delta_{\underline{\xi}} \mathcal{U}=\partial \partial_{u} f\left(u, x^{A}\right)
$$

so that we can use the $u$-dependence in the $f\left(u, x^{A}\right)$ gauge freedom to set $\mathcal{U}_{[0]}=0$, in the notation of (30). Next,

$$
\lim _{r \rightarrow \infty} \delta_{\underline{\xi}} \beta=-\partial_{u} \alpha\left(u, x^{A}\right)+\frac{1}{2} \nsim \bar{\partial} f_{[e]}\left(x^{A}\right)
$$

so that we can use the $u$-dependence in the $\alpha\left(u, x^{A}\right)$ gauge freedom to set $\beta_{[0]}=0$. The remaining gauge freedom is time independent and is determined by $\alpha\left(x^{A}\right)$ and $f\left(x^{A}\right)$. At some fixed retarded time $u=u_{0}$ we have

$$
\lim _{r \rightarrow \infty} \delta_{\underline{\xi}} \mathcal{J}=-\check{\partial}^{2} f\left(x^{A}\right),
$$

which allows us to use the gauge freedom in $f\left(x^{A}\right)$ to set $\mathcal{J}_{[0]}\left(u_{0}, x^{A}\right)=0$. 
In summary, after using gauge freedom, the spin-weight 0 metric fields satisfy

$$
\beta_{[0]}\left(u, x^{A}\right)=0, \quad U_{[0]}\left(u, x^{A}\right)=0, \quad J_{[0]}\left(u_{0}, x^{A}\right)=0,
$$

with the remaining gauge freedom determined by $\alpha\left(x^{A}\right)$.

Now, with these asymptotic conditions on the metric variables along with the asymptotic properties of the matter variables, consider the radial integration of the hypersurface and evolution equations. Given the asymptotic matter conditions $\rho_{r r}=$ $O\left(r^{-4}\right)$ and $P=O\left(r^{-3}\right)$, the integration of the $\beta$-hypersurface equation (25a) and $U$ hypersurface equation (25b) imply $\beta=O\left(r^{-2}\right)$ and $U=O\left(r^{-2}\right)$. As a result, the $J$-evolution equation (26), together with $S=O\left(r^{-2}\right)$, implies

$$
\partial_{u} J_{[0]}\left(u, x^{A}\right)=0
$$

so that, with the initial gauge condition $J_{[0]}\left(u_{0}, x^{A}\right)=0$, we have $J_{[0]}\left(u, x^{A}\right)=0$ at all times. Thus, we can use the gauge freedom to set

$$
\beta=\beta_{[2]} r^{-2}+\ldots, \quad U=U_{[2]} r^{-2}+\ldots, \quad J=J_{[1]} r^{-1}+\ldots,
$$

where the remainder terms are of higher order. Choosing $\xi_{a}=-\nabla_{a} u$ in the formula for the radiation strain (7) gives

$$
\sigma\left(u, x^{A}\right)=\left.\frac{r}{2} q^{A} q^{B} J_{A B}\left(u, r, x^{A}\right)\right|_{r=\infty}=\frac{1}{2} ð^{2} J_{[1]} .
$$

Together with (2) and (9), (47) shows that the spin-weight 0 potential $\Delta \Sigma$ for the radiation memory $\Delta \sigma$ is

$$
\Delta \Sigma=\frac{1}{2} \Delta J_{[1]} .
$$

The remaining gauge freedom (43) of $J$ is according to (39)

$$
\delta_{\underline{\xi}} J_{[1]}\left(u, x^{A}\right)=\alpha\left(x^{A}\right),
$$

so that $J_{[1]}$ may be gauged to 0 in either the limit $u=\infty$ or the limit $u=-\infty$, but the difference $\Delta J_{[1]}$ is gauge invariant.

\section{Weak Asymptotic Stationarity}

The weak asymptotic stationarity condition (see (64)) is key to our analysis of the sky pattern of the memory effect. It serves to control incoming radiation and eliminates two of the three known sources of radiation memory: burst memory and homogeneous wave memory. The third known source, i.e. null memory, depends upon a non-zero integrated energy flux to $\mathcal{I}^{+}$.

9 For the choice $\xi_{A}=-\nabla_{A} u$ in (7) the norm $|\sigma|$ equals the fractional length change $\delta L / L$ measured by a gravitational wave detector. 


\subsection{Notation and useful formulae}

Here we present some useful relations between the flat space Cartesian coordinates $x^{a}=\left(t, x^{i}\right)$, together with the associated orthonormal tetrad

$$
T_{a}=-\partial_{a} t, \quad X_{a}=\partial_{a} x, \quad Y_{a}=\partial_{a} y, \quad Z_{a}=\partial_{a} z
$$

and their spherical analogues. Associated with the spherical coordinate $r$ are the unit vector $r_{a}=\partial_{a} r$ and its the second partial derivative $r_{a b}=\partial_{a} \partial_{b} r$, with the spatial components

$$
r_{i}=\frac{x_{i}}{r}=(\sin \theta \cos \phi, \sin \theta \sin \phi, \cos \theta), \quad r_{i j}=\frac{\delta_{i j}}{r}-\frac{x_{i} x_{j}}{r^{3}},
$$

so that $x^{a}=\left(t, r r^{i}\right)$. With this notation, the second derivative of a function $F(t, r)$ is given by

$\partial_{a} \partial_{b} F=\left(\partial_{t}^{2} F+\frac{\partial_{r} F}{r}\right) T_{a} T_{b}-2\left(\partial_{t} \partial_{r} F\right) T_{(a} r_{b)}+\left(\partial_{r}^{2} F-\frac{\partial_{r} F}{r}\right) r_{a} r_{b}+\left(\frac{\partial_{r} F}{r}\right) \eta_{a b}$.

The vector $Q^{a}$ with $Q^{a} T_{a}=Q^{a} r_{a}=0$ and angular components $Q^{A}=q^{A} / r$ has Cartesian spatial components

$$
Q^{i}=\frac{1}{r} \frac{\partial x^{i}}{\partial x^{A}} q^{A}=(\cos \theta \cos \phi-i \sin \phi, \cos \theta \sin \phi+i \cos \phi,-\sin \theta),
$$

which satisfy

$$
Q_{a} x^{a}=0, Q^{a} \partial_{a} Q^{b}=\frac{\cot \theta}{r} Q^{b}, \quad Q^{i} r_{i j}=\frac{1}{r} Q_{j}, \quad Q^{a} \partial_{a} x^{A}=q^{A} / r
$$

and the useful relations

$$
\begin{aligned}
& \left(Q^{x}\right)^{2}+\left(Q^{y}\right)^{2}=-\sin ^{2} \theta \\
& Q^{x} r_{y}-Q^{y} r_{x}=-i \sin \theta \\
& Q^{x} r_{x}+Q^{y} r_{y}=\sin \theta \cos \theta \\
& Q^{x} r_{y}+Q^{y} r_{x}=\sin \theta\left[2 \cos \theta \cos \phi \sin \phi+i\left(\cos ^{2} \phi-\sin ^{2} \phi\right)\right] \\
& Q_{a} Q_{b}\left(X^{a} Y^{c}-Y^{a} X^{c}\right)\left(X^{b} Y^{d}-Y^{b} X^{d}\right) r_{c} r_{d}=-\sin ^{2} \theta \\
& Q_{a} Q_{b}\left(X^{a} Y^{i}-Y^{a} X^{i}\right)\left(X^{b} Y^{j}-Y^{b} X^{j}\right) \delta_{i j}=-\sin ^{2} \theta
\end{aligned}
$$

\subsection{Weak Asymptotic Stationarity Condition}

5.2.1. Boosted linearized Schwarzschild solution A particle at rest with mass $m$ gives rise to the linearized Schwarzschild metric

$$
g_{a b}=-\left(1-\frac{2 m}{r}\right) T_{a} T_{b}+\left(1+\frac{2 m}{r}\right)\left(X_{a} X_{b}+Y_{a} Y_{b}+Z_{a} Z_{b}\right) .
$$

Burst memory is produced by a particle initially at rest which is later ejected with escape velocity $V$. For a boost in the $z$-direction with four velocity $v^{a}=\Gamma(1,0,0, V)$, with $\Gamma=\left(1-V^{2}\right)^{-1 / 2}$, the resulting memory is

$$
\Delta \sigma=-\frac{2 m \Gamma V^{2} \sin ^{2} \theta}{(V \cos \theta-1)} \text {. }
$$


In order to see how burst memory can be eliminated in a gauge invariant way by a weak asymptotic stationarity condition, consider the corresponding curvature tensor, which for the Schwarzschild solution is determined by the electric part $E_{a b}$ of the Weyl tensor. In the rest frame, $E_{a b} r^{a} r^{b}=2 m / r^{3}$ and $E_{a b} Q^{a} \bar{Q}^{b}=-2 m / r^{3}$ so that

$$
E_{a b}=\frac{m}{r^{3}}\left(3 r_{a} r_{b}-\eta_{a b}-T_{a} T_{b}\right),
$$

where $T^{a}$ is the 4 -velocity of the particle. The corresponding Weyl tensor is

$$
C_{a b c d}=\frac{2 m}{r^{3}}\left[12 r_{[a} T_{b]} r_{[c} T_{d]}+6\left(T_{[a} \eta_{b][c} T_{d]}-r_{[a} \eta_{b][c} r_{d]}\right)-2 \eta_{a[c} \eta_{d] b}\right] .
$$

For a boosted particle with 4 -velocity $T^{a} \rightarrow v^{a}$ and $r$ and $r_{a}$ substituted by the Lorentz covariant expressions

$$
r^{2} \rightarrow R^{2}=x^{a} x_{a}+\left(v_{a} x^{a}\right)^{2}, \quad r_{a} \rightarrow R_{a}=\frac{1}{R}\left[x_{a}+\left(v_{b} x^{b}\right) v_{a}\right]
$$

we obtain

$$
C_{a b c d}=\frac{2 m}{R^{3}}\left[12 R_{[a} v_{b]} R_{[c} v_{c]}+6\left(v_{[a} \eta_{b][c} v_{d]}-R_{[a} \eta_{b][c} R_{d]}\right)-2 \eta_{a[c} \eta_{d] b}\right] .
$$

The corresponding Weyl component $\Psi_{0}$ is

$$
\Psi_{0}=-\frac{3 m}{2 R^{5}}\left[\left(x_{a} K^{a}\right)\left(v_{b} Q^{b}\right)-\left(v_{a} K^{a}\right)\left(x_{b} Q^{b}\right)\right]^{2}
$$

Since $x_{a} Q^{a}=0$ and $x_{a} K^{a}=-u$, we find for a boost in the z-direction with velocity $V$

$$
\Psi_{0}=-\frac{3 m \Gamma^{2} u^{2} V^{2} \sin ^{2} \theta}{2 R^{5}}=-\frac{3 m}{2} \frac{V^{2} \sin ^{2} \theta}{\Gamma^{3}(1+V \cos \theta)^{5 / 2}} \frac{u^{2}}{r^{5}}+O\left(r^{-6}\right) .
$$

For a particle at initially at rest, $\Psi_{0}=0$ and $\Psi_{0}$ satisfies (63) after it is ejected. Thus the ejection and the associated burst memory can be eliminated by requiring the weak stationarity condition

$$
0=\Delta \lim _{r \rightarrow \infty} r^{5} \partial_{u}^{2} \Psi_{0}
$$

This also rules out the memory effect due to the time reversed process of the capture of particles incident from infinity.

Note that (64) does not restrict higher order boosted multipoles, whose Weyl curvature $\Psi_{0}$ falls off faster than $1 / r^{5}$. However, as we show next, this weak asymptotic stationarity condition does rule out the memory effect due to source-free waves. Expressed in terms of the metric variable $J$ by means of (28), weak asymptotic stationarity (64) restricts the metric coefficient $J_{[3]}$ according to

$$
0=\Delta \partial_{u}^{2} J_{[3]}
$$

In particular, this places no restriction on the time dependence of the radiation field $J_{[1]}$, and therefore it is not a direct restriction on the gravitational memory $\Delta \Sigma$. 
5.2.2. Source-free gravitational waves Here we consider the standard formalism [31] for linearized metric perturbations $h_{a b}$ of a Minkowski background metric,

$$
g_{a b}=\eta_{a b}+h_{a b}, \quad g^{a b}=\eta^{a b}-h^{a b}, \quad \operatorname{det}\left(g_{a b}\right)=-1+h_{a}^{a}
$$

where indices are raised and lowered with the Minkowski metric. The densitized version of the perturbation is given by

$$
\sqrt{-g} g_{a b}=\eta_{a b}+\gamma_{a b}
$$

where

$$
\gamma_{a b}=h_{a b}-\frac{1}{2} \eta_{a b} h_{c}^{c}
$$

We adopt the harmonic gauge condition $\partial_{b} \gamma^{a b}=0$, in which the source-free linearized Einstein equations take the simple form $\eta^{a b} \partial_{a} \partial_{b} \gamma_{c d}=0$.

We construct source free linearized waves in the harmonic gauge by using the gravitational analogue of a Hertz potential $H^{a c b d}$ [44, 43], which has the symmetries

$$
H^{a c b d}=H^{[a c] b d}=H^{a c[b d]}=H^{b d a c}
$$

and satisfies the flat space wave equation $\partial^{e} \partial_{e} H^{a c b d}=0$. As a result, the densitized metric perturbation

$$
\gamma^{a b}=\partial_{c} \partial_{d} H^{a c b d}
$$

satisfies the linearized Einstein equations in the harmonic gauge.

Source-free, ingoing-outgoing gravitational waves can be generated from the potential

$$
H^{a c b d}=K^{a c b d} \frac{f(t-r)-f(t+r)}{r}, \partial_{e} K^{a b c d}=0,
$$

which gives rise to the perturbation

$$
\gamma^{a b}=K^{a c b d} \partial_{b} \partial_{c} \frac{f(t-r)-f(t+r)}{r} .
$$

According to (47), the strain of the radiation field on $\mathcal{I}^{+}$is given by

$$
\sigma=\lim _{r \rightarrow \infty} \frac{1}{2} r Q_{a} Q_{b} \gamma^{a b}
$$

For these vacuum solutions of the field equations $R_{a b c d}=C_{a b c d}$ and the Weyl component $\Psi_{0}$ is

$$
\Psi_{0}=-\frac{1}{4} R_{a b c d} K^{a} Q^{b} K^{c} Q^{d}=-\frac{1}{8}\left(2 \gamma_{a b, c d}-\gamma_{a c, b d}-\gamma_{b d, a c}\right) K^{a} Q^{b} K^{c} Q^{d},
$$

where the individual terms are determined by the tetrad components of $\gamma_{a b}$ according to

$$
\begin{aligned}
& \gamma_{a b, c d} Q^{a} Q^{b} K^{c} K^{d}=K^{c} \partial_{c}\left[K^{d} \partial_{d}\left(\gamma_{a b} Q^{a} Q^{b}\right)\right] \\
& \gamma_{a b, c d} Q^{a} K^{b} Q^{c} K^{d}=K^{c} \partial_{c}\left[Q^{d} \partial_{d}\left(\frac{1}{\sin \theta} \gamma_{a b} Q^{a} K^{b}\right) \sin \theta-\frac{1}{r} \gamma_{a b} Q^{a} Q^{b}\right] \\
& \gamma_{a b, c d} K^{a} K^{b} Q^{c} Q^{d}=Q^{c} \partial_{c}\left[\frac{Q^{d} \partial_{d}\left(\gamma_{a b} K^{a} K^{b}\right)}{\sin \theta}\right] \sin \theta-\frac{4 \sin \theta}{r} Q^{c} \partial_{c}\left(\frac{\gamma_{a b} Q^{a} K^{b}}{\sin \theta}\right)+\frac{2}{r^{2}} \gamma_{a b} Q^{a} Q^{b} .
\end{aligned}
$$


Considering $\gamma_{a b} Q^{a} Q^{b}, \gamma_{a b} K^{a} Q^{b}$ and $\gamma_{a b} K^{a} K^{b}$ to be functions of $(u, r, \theta, \phi)$, we rewrite (75a) $-(75 c)$ as

$$
\begin{aligned}
& \gamma_{a b, c d} Q^{a} Q^{b} K^{c} K^{d}=\partial_{r}^{2}\left(\gamma_{a b} Q^{a} Q^{b}\right), \\
& \gamma_{a b, c d} Q^{a} K^{b} Q^{c} K^{d}=\partial_{r}\left[\frac{\sin \theta}{r} q^{A} \partial_{A}\left(\frac{\gamma_{a b} Q^{a} K^{b}}{\sin \theta}\right)\right]-\partial_{r}\left[\frac{\gamma_{a b} Q^{a} Q^{b}}{r}\right], \\
& \gamma_{a b, c d} K^{a} K^{b} Q^{c} Q^{d}=\frac{\sin \theta}{r^{2}} q^{A} \partial_{A}\left[\frac{q^{A} \partial_{A}\left(\gamma_{a b} K^{a} K^{b}\right)}{\sin \theta}-\frac{4 \gamma_{a b} Q^{a} K^{b}}{\sin \theta}\right]+\frac{2}{r^{2}} \gamma_{a b} Q^{a} Q^{b} .
\end{aligned}
$$

For our purpose, it suffices to consider the perturbation (72) determined by

$$
f(\tau)=\left\{\begin{array}{clc}
\frac{1}{2} C \tau^{2} & : & T<\tau \\
F(\tau) & : & 0 \leq \tau \leq T \\
0 & : & \tau<0
\end{array}\right.
$$

where $F(\tau)$ is chosen to make the solution smooth. In particular,

$$
\begin{array}{ll}
f^{\prime \prime}(u)=C, \quad f^{\prime \prime}(v)=C, & : u>T \\
f^{\prime \prime}(u)=0, \quad f^{\prime \prime}(v)=C, & : u<0 \text { and } u+2 r>T .
\end{array}
$$

\subsubsection{Quadrupole B-mode gravitational wave memory The choice}

$$
K^{a b c d}=\frac{1}{4}\left(T^{[a} Z^{b]} X^{[c} Y^{d]}+X^{[a} Y^{b]} T^{[c} Z^{d]}\right)
$$

in (72), along with the help of (152), gives rise to the purely B-mode quadrupole perturbation with components

$$
\begin{aligned}
Q^{\alpha} Q^{\beta} \gamma_{\alpha \beta} & =2 i \sin ^{2} \theta\left(\frac{f^{\prime \prime}(u)+f^{\prime \prime}(u+2 r)}{r}+\frac{f^{\prime}(u)-f^{\prime}(u+2 r)}{r^{2}}\right), \\
K^{\alpha} Q^{\beta} \gamma_{\alpha \beta} & =i \cos \theta \sin \theta\left(\frac{-2 f^{\prime \prime}(u+2 r)}{r}+\frac{2 f^{\prime}(u)+4 f^{\prime}(u+2 r)}{r^{2}}\right. \\
& \left.+\frac{3 f(u)-3 f(u+2 r)}{r^{3}}\right), \\
K^{\alpha} K^{\beta} \gamma_{\alpha \beta} & =0,
\end{aligned}
$$

corresponding to spin-weight $(l=2, m=0)$ spherical harmonics. This perturbation gives rise to the $\mathrm{B}$-mode radiation strain

$$
\sigma_{[b]}\left(u, x^{A}\right)=-i \sin ^{2} \theta \lim _{r \rightarrow \infty}\left[f^{\prime \prime}(u)+f^{\prime \prime}(u+2 r)\right] .
$$

The waveform (77) then leads to the non-zero radiation memory

$$
\Delta \sigma_{[b]}=-i C \sin ^{2} \theta .
$$

Thus homogeneous waves with non-zero B-mode radiation memory exist.

In order to show that weak asymptotic stationarity rules out these waves we calculate the Weyl component $\Psi_{0}$. Substitution of $(\underline{80 a})-(\underline{80 c})$ into $(76 a)-(76 c)$ yields the required second derivatives of the metric,

$\gamma_{a b, c d} Q^{a} Q^{b} K^{a} K^{b}=i \sin ^{2} \theta\left\{\frac{8 f^{\prime \prime \prime \prime}(u+2 r)}{r}-\frac{16 f^{\prime \prime \prime}(u+2 r)}{r^{2}}+\frac{4 f^{\prime \prime}(u)+20 f^{\prime \prime}(u+2 r)}{r^{3}}\right.$ 


$$
\begin{gathered}
\left.\quad+\frac{12\left[f^{\prime}(u)-f^{\prime}(u+2 r)\right]}{r^{4}}\right\}, \\
\gamma_{a b, c d} Q^{a} K^{b} Q^{c} K^{d}=i \sin ^{2} \theta\left\{\frac{4\left[f^{\prime \prime}(u)-f^{\prime \prime}(u+2 r)\right]}{r^{3}}+\frac{12\left[f^{\prime}(u)+f^{\prime}(u+2 r)\right]}{r^{4}}\right. \\
\left.+\frac{12[f(u)-f(u+2 r)]}{r^{5}}\right\}, \\
\gamma_{a b, c d} K^{a} K^{b} Q^{c} Q^{d}=i \sin ^{2} \theta\left\{\frac{4\left[f^{\prime \prime}(u)-f^{\prime \prime}(u+2 r)\right]}{r^{3}}+\frac{12\left[f^{\prime}(u)+f^{\prime}(u+2 r)\right]}{r^{4}}\right. \\
\left.+\frac{12[f(u)-f(u+2 r)]}{r^{5}}\right\} .
\end{gathered}
$$

The subsequent evaluation of (74) leads to

$$
\begin{aligned}
\Psi_{0[b]}:=\Psi_{0}=i \sin ^{2} \theta\{ & \frac{f^{\prime \prime \prime \prime}(u+2 r)}{r}-\frac{2 f^{\prime \prime \prime}(u+2 r)}{r^{2}}+\frac{3 f^{\prime \prime}(u+2 r)}{r^{3}}-\frac{3 f^{\prime}(u+2 r)}{r^{4}} \\
& \left.-\frac{3}{2} \frac{f(u)-f(u+2 r)}{r^{5}}\right\} .
\end{aligned}
$$

For the waveform (77), we find

$$
\begin{aligned}
& \Psi_{0[b]}=0, \quad u>T, \\
& \Psi_{0[b]}=\frac{3 i C u^{2} \sin ^{2} \theta}{4 r^{5}}, \quad u<0, u+2 r>T .
\end{aligned}
$$

Consequently, the weak asymptotic stationarity condition (64) implies that $C=0$ and rules out the B-mode memory (82) arising from homogeneous ingoing-outgoing waves.

5.2.4. Quadrupole E-mode gravitational wave memory Ingoing-outgoing waves for electric type homogeneous waves can be generated from the dual * $K^{a b c d}$ of (79),

$$
{ }^{*} K^{a c b d}=\frac{1}{2} \eta^{a b}{ }_{e f} K^{e f c d}=\frac{1}{3}\left(\eta^{a b} \eta^{c d}-\eta^{a d} \eta^{b c}\right)+\frac{1}{4} T^{[a} Z^{c]} T^{[b} Z^{d]}-\frac{1}{4} X^{[a} Y^{c]} X^{[b} Y^{d]}
$$

The previous procedure for the magnetic type gravitational waves, using ${ }^{*} K^{a b c d}$ instead of $K^{a b c d}$, leads to the quadrupole E-mode gravitational memory related to the B-mode memory (82) by

$$
\Delta \sigma_{[e]}=-i \Delta \sigma_{[b]}=C \sin ^{2} \theta
$$

(also compare with (11)).

Similarily, we find $\Psi_{0[e]}=-i \Psi_{0[b]}$, so, referring to (85),

$$
\begin{aligned}
& \Psi_{0[e]}=0, \quad u>T, \\
& \Psi_{0[e]}=\frac{3 C u^{2} \sin ^{2} \theta}{4 r^{5}}, \quad u<0, u+2 r>T .
\end{aligned}
$$

Consequently, the weak asymptotic stationarity condition (64) again requires $C=0$ and rules out E-mode memory arising from homogeneous ingoing-outgoing waves. 


\section{Gravitational Memory}

We now discuss our central issue, the global analysis of the $\mathrm{E}$ and B-mode patterns of the linearized gravitational memory. For this purpose, we consider the general asymptotic solution of the linearized Einstein equations at $\mathcal{I}^{+}$and, in particular, the limiting behavior at $u= \pm \infty$, subject to the asymptotic conditions on the matter and metric discussed in Sec. 4 ,

The solution of the Einstein equations, as detailed in Sec. 3, assumes an asymptotic expansion of the matter and metric variables in terms of a $1 / r$ expansion. For the metric variables, we assume, in accord with (46), that the strain variable $J$ has the expansion

$$
J=J_{[1]} r^{-1}+J_{[2]} r^{-2}+J_{[3]} r^{-3}+\text { higher order terms , }
$$

and, for the matter fields, we assume in accord with (B3a) $-(33 \mathrm{C})$

$$
\rho_{r r}=\frac{\rho_{r r[4]}}{r^{4}}+\ldots, \quad P=\frac{P_{[3]}}{r^{3}}+\ldots, \quad S_{0}=\frac{S_{0[2]}}{r^{2}}+\ldots, \quad S=\frac{S_{[2]}}{r^{2}}+\ldots .
$$

We proceed to use these expansions to integrate the linearized equations.

The integration of the $\beta$-hypersurface equation gives the asymptotic dependence

$$
\beta=-\frac{\kappa \rho_{r r[4]}}{8 r^{2}}+O\left(1 / r^{3}\right)
$$

and together with $(\underline{33 d})$

$$
\partial_{u} \beta_{[2]}=0, \quad u= \pm \infty .
$$

Integration of the $U$-hypersurface equation $(25 b)$ yields

$$
\begin{aligned}
& U=\frac{U_{[2]}}{r^{2}}+\frac{U_{[3]}}{r^{3}}+\left(-\frac{1+3 \ln r}{9 r^{3}}\right) U_{[l o g]}+\text { higher order terms }, \\
& U_{[2]}=-\frac{1}{4}(ð \bar{\delta}+2) J_{[1]}, \\
& U_{[3]}=-\frac{L}{3}, \\
& U_{[l o g]}=(ð \bar{\varnothing}+2) J_{[2]}-8 \beta_{[2]}+2 \kappa P_{[3]},
\end{aligned}
$$

where the real and imaginary parts of the function of integration $L\left(u, x^{A}\right)$, appearing in $U_{[3]}$ are, respectively, the dipole-moment aspect and the angular-momentum aspect. Inserting the asymptotic solutions for $\beta$ and $U$ into the evolution equation (26) yields

$$
\begin{aligned}
& 0=\partial_{u} J_{[2]}, \\
& 0=U_{[l o g]}=(ð \bar{\partial}+2) J_{[2]}-8 \beta_{[2]}+2 \kappa P_{[3]}, \\
& 0=2 \partial_{u} J_{[3]}+J_{[2]}+U_{[3]}+2 \beta_{[2]}+\kappa S_{[2]} .
\end{aligned}
$$

As a consequence of (94b), the logarithmic term in (93a $)$ vanishes, i.e.

$$
U=\frac{U_{[2]}}{r^{2}}+\frac{U_{[3]}}{r^{3}}+\text { higher order terms, }
$$


which is consistent with asymptotic expansion in $1 / r$ required by the peeling property. Subsequently, integration of the $W$ - hypersurface equation (25c), while using (95), gives

$$
\begin{aligned}
& W=\frac{W_{[1]}}{r}+\frac{W_{[2]}}{r^{2}}+\ldots, \\
& W_{[1]}:=-2 M, \\
& W_{[2]}:=-\frac{1}{2} \overline{\widetilde{\partial}} U_{[e 1]}-(ð \bar{ð}+2) \beta_{[2]}+\frac{\kappa}{2}\left(ð \bar{ð} P_{[e 3]}+S_{0[2]}\right),
\end{aligned}
$$

where the function of integration $M=M\left(u, x^{A}\right)$ is the mass aspect, normalized so that the $u u$-component of the metric perturbation equals $2 M / r$ in the static case. This general solution must also obey the supplementary conditions at some radius $r$. In the next two sections, we impose these conditions in the limit of $\mathcal{I}^{+}$, where their real and imaginary parts constrain the $E$ and $B$-mode memory, respectively. The matter terms which appear in the spin-weight 0 version $(\underline{27 a})$ and $(\underline{27 b})$ are expanded according to $(33 a)$ and $(33 b)$ as

$$
\rho_{u u}=\frac{\rho_{u u[2]}}{r^{2}}+\frac{\rho_{u u[3]}}{r^{3}}+\frac{\rho_{u u[4]}}{r^{4}}+\ldots, \quad N=\frac{N_{[2]}}{r^{2}}+\frac{N_{[3]}}{r^{3}}+\ldots .
$$

\subsection{Implications for the E-mode gravitational memory}

The supplementary condition (27a), i.e. $R_{u u}=\kappa \rho_{u u}$, is purely real, whereas (27b), resulting from the spin-weight 0 version of $q^{A} R_{u A}=\kappa \mathcal{N}$, has both a real and imaginary part. In order to constrain the E-mode memory we consider the real part of (27b) ,

$$
0=\partial_{u}\left[\frac{1}{4}(\bar{\varnothing} \partial+2) J_{[e]}-\beta-\frac{1}{2} r^{2} \partial_{r} U_{[e]}\right]+\frac{1}{2} \partial_{r} W+\frac{1}{2} \partial_{r}^{2}\left(r^{2} U_{[e]}\right)-\kappa N_{[e]} .
$$

Substitution of the asymptotic expansions for $\beta, W$, and the real parts $J_{[e]}$ and $U_{[e]}$, yields the leading order terms

$$
\begin{aligned}
& \left.\left[r^{-2} \text { part of (27a) }\right)\right] 0=\partial_{u} W_{[1]}-\partial_{u} \partial \bar{\partial} U_{[e 2]}-\kappa \rho_{u u[2]}, \\
& {\left[r^{-1} \text { part of (98) }\right] 0=\partial_{u}(\widetilde{\partial} \bar{\gamma}+2) J_{[e 1]}+4 \partial_{u} U_{[e 2]} \text {, }} \\
& {\left[r^{-2} \text { part of (98) }\right] 0=\partial_{u}(ð \bar{\gamma}+2) J_{[e 2]}+6 \partial_{u} U_{[e 3]}-4 \partial_{u} \beta_{[2]}-2 W_{[1]}-4 \kappa N_{[e 2]} \text {. }}
\end{aligned}
$$

Substitution of the electric part of $U$ from (93b) into (99b) shows that (99b) is already satisfied. Using the formulae (9) and (47) for the strain, along with (93b) and (96b), the supplementary condition (99a) implies

$$
\partial_{u} M=\frac{1}{4} \partial_{u} \precsim \bar{\partial}(ð \bar{\varnothing}+2) \Sigma_{[e]}-\frac{\kappa}{2} \rho_{u u[2]} .
$$

Integration over retarded time from $u=-\infty$ to $u=\infty$ gives

$$
\Delta ð \bar{\partial}(ð \bar{\partial}+2) \Sigma_{[e]}=4 \Delta M+2 \kappa \int_{-\infty}^{+\infty} \rho_{u u[2]} d u \text {. }
$$

Here the contribution from $\rho_{u u[2]}$ is the null memory arising from the net transport of energy to infinity by the matter. In the absence of null memory, we thus obtain that the E-mode memory $\Delta \Sigma_{[e]}$ is related to the change in the mass aspect $\Delta M$. 
Next, the supplementary condition (99c), along with the electric part of (94b), gives

$$
0=2 \kappa \partial_{u} P_{[e 3]}-4 \partial_{u} \beta_{[2]}-6 \partial_{u} U_{[e 3]}+2 W_{[1]}+4 \kappa N_{[e 2]} .
$$

Differencing (102) between $u= \pm \infty$, while employing the matter conditions (33c) and $(\underline{3 d d})$, the condition (92), and the relations (93c) and (96b), gives

$$
\Delta \partial_{u} L_{[e]}=2 \Delta M .
$$

Consequently (101) shows that the electric memory $\Delta \Sigma_{[e]}$ can alternatively be expressed in terms of the dipole-moment aspect $L_{[e]}$ by

$$
\Delta \check{\partial} \bar{\partial}(\widetilde{\partial} \bar{\partial}+2) \Sigma_{[e]}=2 \Delta \partial_{u} L_{[e]}+2 \kappa \int_{-\infty}^{+\infty} \rho_{u u[2]} d u .
$$

Next, application of the weak asymptotic stationarity condition (65) to the $u$ derivative of (94c), together with (92), (93c) and (94a), gives

$$
\Delta \partial_{u} L_{[e]}=3 \kappa \Delta \partial_{u} S_{[e 2]} .
$$

As a result, (104) relates the E-mode memory to the net change in the $u$-derivative of the anisotropic stress according to

$$
\Delta ð \bar{\delta}(ð \bar{\partial}+2) \Sigma_{[e]}=6 \kappa \Delta \partial_{u} S_{[e 2]}+2 \kappa \int_{-\infty}^{+\infty} \rho_{u u[2]} d u .
$$

Thus, except for the null memory arising from $\rho_{u u[2]}$, the only memory effect allowed by the weak asymptotic stationarity condition must arise from a matter distribution with the asymptotic behavior $\Delta \partial_{u} S_{[e 2]} \neq 0$. A detailed discussion of this possibility is postponed to Sec. 6.3,

\subsection{Implications for the B-mode gravitational memory}

As seen from (48), the B-mode gravitational memory $\Delta \Sigma_{[b]}$ is determined by the imaginary part $J_{[b 1]}$. As only the fields $J, U, P, N$ and $S$ have imaginary parts, the only relevant supplementary equation is the imaginary part of (27b),

$$
0=\partial_{u}\left[\frac{1}{4}(\bar{\varnothing} ð+2) J_{[b]}-\frac{1}{2} r^{2} \partial_{r} U_{[b]}\right]+\frac{1}{2} \partial_{r}^{2}\left(r^{2} U_{[b]}\right)+\frac{1}{2} \bar{\varnothing} \check{\partial} U_{[b]}-\kappa N_{[b]} .
$$

The leading two orders of (107), along with the imaginary parts of (89), (95) and (97), give

$$
\begin{array}{ll}
\left(r^{-1}\right): & 0=\frac{1}{4} \partial_{u}(\bar{\varnothing} \partial+2) J_{[b 1]}+\partial_{u} U_{[b 2]}, \\
\left(r^{-2}\right): & 0=\frac{1}{4} \partial_{u}(\bar{\varnothing} \partial+2) J_{[b 2]}+\frac{3}{2} \partial_{u} U_{[b 3]}+\frac{1}{2} \bar{\varnothing} \partial U_{[b 2]}-\kappa N_{[b 2]} .
\end{array}
$$

The imaginary part of (93b) implies that (108a) is already satisfied. From the imaginary parts of (33c), (93b), (93c) and (94a) the second condition (108b) simplifies at $u= \pm \infty$ to

$$
0=\frac{1}{4} \bar{\partial} \partial(\bar{\partial} \partial+2) J_{[b 1]}+\partial_{u} L_{[b]},
$$


so that

$$
\Delta \bar{\varnothing} ð(\bar{ð} ð+2) \Sigma_{[b]}=-2 \Delta \partial_{u} L_{[b]} \cdot
$$

Thus the B-mode memory $\Delta \Sigma_{[b]}$ is related to the angular momentum aspect $L_{[b]}$ in a similar way that the E-mode memory is related to the dipole-moment aspect $L_{[e]}$. Also note, there is no analogue of the null memory for the B-mode case. As in the E-mode case, the B-mode memory can be related to the asymptotic anisotropic stress $S$ by applying the asymptotic stationarity condition (65) to the magnetic part of (94c) together with (93c) and (94a),

$$
\Delta \overline{\bar{\partial}}(\bar{ð} \check{\partial}+2) \Sigma_{[b]}=-6 \kappa \Delta \partial_{u} S_{[b 2]} \cdot
$$

Thus, after using weak asymptotic stationarity to rule out the known source of B-mode memory, i.e. a homogeneous wave, a non-compact matter distribution with the asymptotic stress $\Delta \partial_{u} S_{[b 2]} \neq 0$ remains the only possibility.

\subsection{Matter implications for $E$ and B-mode gravitational memory}

From (106) and (111), we have shown that the weak asymptotic stationarity condition condition leads to

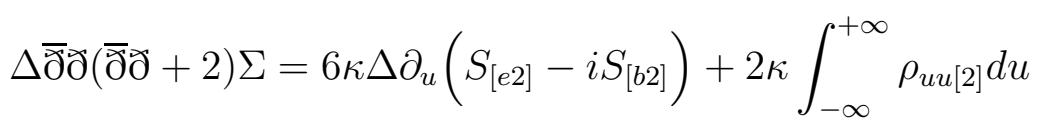

In the absence of null memory $\int_{-\infty}^{+\infty} \rho_{u u[2]} d u=0$ so that (112) reduces to

$$
\Delta \bar{\varnothing} ð(\bar{ð} \check{\partial}+2) \Sigma=6 \kappa \Delta \partial_{u}\left(S_{[e 2]}-i S_{[b 2]}\right),
$$

which shows that all remaining possible sources of gravitational memory are determined by the retarded time derivatives of the stress $\partial_{u} S_{[2]}$ at $u= \pm \infty$. Recall that $\Sigma$ contains no monopole or dipole harmonics which otherwise would be allowed by the inverse of the $\bar{\varnothing} \precsim(\bar{\precsim} \partial+2)$ operator. Since the matter stresses $S$ were defined in Sec. 3 with respect to a most general energy momentum tensor and without specification of the type of matter, we now discuss the asymptotic behavior of $\partial_{u} S_{[2]}$ for various sources.

Sources of compact support: For matter which vanishes in a neighborhood of $\mathcal{I}^{+}$at all finite $u$, it immediately follows that $\partial_{u} S_{[2]}=0$ for all $u$. Note that this does not rule out matter confined inside an expanding (or contracting) worldtube $r=R(u)$, where $R(u) \rightarrow \infty$ as $u \rightarrow \pm \infty$.

Scalar fields: The stress-energy tensor of a scalar field $\Phi$ with mass $m$,

$$
T_{a b}=\left(\partial_{a} \Phi\right)\left(\partial_{b} \Phi\right)-\frac{1}{2} \eta_{a b}\left[\eta^{c d}\left(\partial_{c} \Phi\right)\left(\partial_{d} \Phi\right)+\frac{m^{2}}{\hbar^{2}} \Phi^{2}\right]
$$

gives rise to the anisotropic stress

$$
\check{\partial}^{2} S=(ð \Phi)^{2}
$$

For a massive scalar field on a Minkowski background, it has been shown that $\Phi$ falls off faster than any finite power $1 / r^{n}$ at $\mathcal{I}^{+}$[41, 42]. Therefore $S_{2}$ vanishes for all $u$. 
For a massless scalar field, the asymptotic radiative behavior $\Phi\left(x^{a}\right)=$ $\Phi_{[1]}\left(u, x^{A}\right) r^{-1}+O\left(r^{-2}\right)$ implies

$$
T_{u}^{r}=-\frac{1}{r^{2}}\left(\partial_{u} \Phi_{[1]}\right)^{2}+\ldots \quad, \quad \partial^{2} \partial_{u} S_{[2]}=2\left(ð \Phi_{[1]}\right) \partial \partial_{u} \Phi_{[1]} .
$$

The requirement that the $u$-integration of the energy flux $T_{u}^{r}$ to $\mathcal{I}^{+}$be finite implies that $\partial_{u} \Phi_{[1]}=0$ for $u= \pm \infty$. Thus $\Delta \partial_{u} S_{[2]}=0$.

Electromagnetic fields: The stress-energy tensor of an electromagnetic field $F_{a b}$,

$$
T_{a b}=F_{a c} F_{b}^{c}-\frac{1}{4} \eta_{a b} F^{c d} F_{c d}
$$

gives rise to an anisotropic stress

$$
\check{\partial}^{2} S=q^{A} q^{B} F_{A c} F_{B}^{c}
$$

The asymptotic behavior of the electromagnetic field, dictated by the peeling property implies (in null spherical coordinates) $q^{A} F_{A u}=O(1), q^{A} \bar{q}^{B} F_{A B}=O(1), F_{u r}=O\left(1 / r^{2}\right)$ and $q^{A} F_{A r}=O\left(1 / r^{2}\right)$. As a result,

$$
T_{u}^{u}=\frac{1}{r^{2}}\left|q^{A} F_{A u[0]}\right|^{2}+\ldots, \quad \check{\partial}^{2} S_{[2]}=-2 q^{A} q^{B} F_{A u[0]} F_{B r[2]} .
$$

As in the scalar case, the requirement that the $u$-integration of the energy flux $T_{u}^{r}$ to

$\mathcal{I}^{+}$be finite implies that $q^{A} F_{A u[0]}=0$ for $u= \pm \infty$. Thus $S_{[2]}=0$ at $u= \pm \infty$ and consequently $\Delta \partial_{u} S_{[2]}=0$.

Perfect fluid halo: The stress-energy tensor of a perfect fluid, with matter density $\rho$, pressure $p$ and 4-velocity $v^{a}$,

$$
T_{a b}=(\rho+p) v_{a} v_{b}+p \eta_{a b},
$$

gives rise to the anisotropic stress

$$
\varlimsup^{2} S=(\rho+p)\left(q^{A} v_{A}\right)^{2}=r^{4}(\rho+p)\left(q_{A} v^{A}\right)^{2} .
$$

Here, the normalization $v_{a} v^{a}=-1$ requires $\left.v^{u}\right|_{r=\infty} \neq 0, v^{r}=O(1)$ and $v^{A}=O(1 / r)$. The finite energy-momentum conditions, $T_{u}^{u}=O\left(1 / r^{4}\right)$ and $T_{r}^{u}=O\left(1 / r^{4}\right)$, imply $\rho=O\left(1 / r^{4}\right)$ and $p=O\left(1 / r^{4}\right)$ and consequently

$$
\check{\partial}^{2} \partial_{u} S_{[2]}=q_{A} q_{B} \partial_{u}\left[\left(\rho_{[4]}+p_{[4]}\right) v_{[1]}^{A} v_{[1]}^{B}\right] \text {. }
$$

The leading terms in the $A$-component of the conservation laws (32) imply at $u= \pm \infty$ that

$$
0=\partial_{u}\left[\left(\rho_{[4]}+p_{[4]}\right) v_{[1]}^{A} v_{[0]}^{u}\right] .
$$

The equations of motion $\left(\delta^{a}{ }_{b}+v^{a} v_{b}\right) \nabla_{c} T^{c b}=0$ give to leading order in $1 / r$

$$
\begin{aligned}
& \left(\rho_{[4]}+p_{[4]}\right) \partial_{u} v_{[0]}^{u}+v_{[0]}^{u} \partial_{u} p_{[4]}=0, \\
& \left(\rho_{[4]}+p_{[4]}\right) v_{[0]}^{u} \partial_{u} v_{[0]}^{r}+\left(-1+v_{[0]}^{r} v_{[0]}^{u}\right) \partial_{u} p_{[4]}=0, \\
& \left(\rho_{[4]}+p_{[4]}\right) \partial_{u} v_{[1]}^{A}+v_{[1]}^{A} \partial_{u} p_{[4]}=0 .
\end{aligned}
$$


We have

$$
\begin{aligned}
\check{\partial}^{2} v_{[0]}^{u} \partial_{u} S_{[2]}= & q_{A} q_{B} v_{[0]}^{u} \partial_{u}\left[\left(\rho_{[4]}+p_{[4]}\right) v_{[1]}^{A} v_{[1]}^{B}\right] \\
=-q_{A} q_{B} & \left\{v_{[0]}^{u}\left(\partial_{u} v_{[1]}^{A}\right)\left(\rho_{[4]}+p_{[4]}\right) v_{[1]}^{B}+v^{A} \partial_{u}\left[\left(\rho_{[4]}+p_{[4]}\right) v_{[1]}^{B} v_{[0]}^{u}\right]\right. \\
& \left.-\left(\partial_{u} v_{[0]}^{u}\right)\left(\rho_{[4]}+p_{[4]}\right) v_{[1]}^{A} v_{[1]}^{B}\right\} .
\end{aligned}
$$

As a result, a direct application of (123), (124), and (126) gives, after some algebra,

$$
\check{\partial}^{2} v_{[0]}^{u} \partial_{u} S_{[2]}=0, \quad u= \pm \infty .
$$

Therefore $\Delta \partial_{u} S_{2}=0$ for a perfect fluid halo.

\section{Summary}

In the context of linearized theory, we have shown that the weak asymptotic stationarity condition, along with standard asymptotic conditions on the metric and matter stressenergy tensor, imply that the only possible sources of gravitational radiation memory, other than null memory, require matter with asymptotic stress satisfying $\Delta \partial_{u} S_{2} \neq 0$. While, on the basis of general principles, we cannot rule out such an anisotropic, time dependent stress, we have shown that it is ruled out by common sources including matter confined to an expanding or contracting worldtube, scalar fields, electromagnetic fields and a perfect fluid halo. Barring exotic matter sources, our results imply that the E-mode memory effect is restricted to null memory and the two known sources that are eliminated by the weak asymptotic stationarity condition, i.e. burst memory (and its time reversed counterpart) and homogeneous wave memory. The results for Bmode memory are more restrictive since there is no B-mode analogue to null memory or burst memory. Thus the only known source of B-mode memory appears to be of primordial origin, corresponding in the linearized theory to a source free, ingoingoutgoing gravitational wave entering from past null infinity.

Although our results are based upon linearized theory, it is straightforward to extend the underlying approach, an asymptotic expansion of the Bondi-Sachs metric along the outgoing null cones, to the nonlinear theory. Thus our results set the stage for an investigation of how the presence of black holes affect the sky pattern of the memory effect.

\section{Acknowledgments}

T.M is grateful for hospitality of the AEI in Golm where this project was initiated and appreciates valuable discussions and support from P. Jofré, Q. Kral, A. Bonsor, C. Malone and A. J. Penner. J.W. was supported by NSF grant PHY-1505965 to the University of Pittsburgh. 


\section{Appendix A. Global electromagnetic memory revisited}

Here we revisit the global electromagnetic memory analysis and show that a weaker asymptotic stationarity condition than the one employed in [13] yields the same results. With a slight change of notation from [13], we adopt the spin-weight formulation analogous to the linearized gravitational case. In this notation, the Maxwell field and vector potential, $F_{a b}=2 \partial_{[a} A_{b]}$, have angular components in null spherical coordinates represented by the spin-weight 0 potentials $\mathscr{E}$ and $\mathscr{A}$ according to

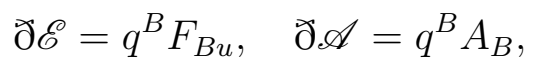

where, by convention, $\mathscr{E}$ and $\mathscr{A}$ have no $l=0$ component. We adopt a null gauge $A_{r}=0$, which is the electromagnetic analogue of the Bondi-Sachs gauge [13, 35]. We use the remaining gauge freedom, $A_{a} \rightarrow A_{a}+\partial_{a} \Lambda\left(x^{a}\right)$, to set $A_{u[0]}=\left.A_{u}\right|_{\mathcal{I}^{+}}=0$. The remnant gauge freedom $A_{B} \rightarrow A_{B}+\partial_{B} \Lambda\left(x^{C}\right)$ is the electromagnetic analogue of a BMS supertranslation. The electromagnetic radiation memory for a test particle with unit charge and mass is

$$
\Delta \partial \mathscr{V}:=\lim _{r \rightarrow \infty} \int_{-\infty}^{\infty} \check{\partial} \mathscr{E} d u=-\Delta \partial \mathscr{A}_{[0]}
$$

The peeling property of an isolated system requires that the electromagnetic Newman-Penrose component $\Phi_{0}:=\frac{1}{2} F_{a b} K^{a} Q^{b}=O\left(1 / r^{3}\right)$. The new weak asymptotic stationarity condition is

$$
\Delta \lim _{r \rightarrow \infty} r^{3} \partial_{u} \Phi_{0}=\Delta \partial_{u} \Phi_{0[3]}=-\Delta \partial_{u} \partial^{\prime} \mathscr{A}_{[1]}=0 .
$$

There are three known causes of electromagnetic memory: burst memory due to the ejection of charged particles, homogeneous wave memory due to source free waves and null memory due to the flow of charge to $\mathcal{I}^{+}$by a (hypothetical) charged massless field or fluid. We now show that the weak asymptotic stationarity condition (A.3) rules out burst memory and homogeneous wave memory.

Burst memory results from the asymptotic behavior of a boosted Coulomb field, which can be described in the Lorentz covariant form

$$
A_{a}=\frac{q}{R} v_{a}, \quad F_{a b}=\frac{q}{R^{3}}\left(x_{a} v_{b}-x_{b} v_{a}\right),
$$

where $v_{a}$ the four velocity of a particle with charge $q$ and $R$ is given by (60). A charged particle which is initially at rest and ejected with velocity $V$ in the $z$-direction gives rise to the non-zero burst memory

$$
ð \Delta \mathscr{V}=\frac{q V \sin \theta}{1-V \cos \theta} .
$$

The resulting electromagnetic Newman-Penrose scalar is

$$
\Phi_{0}=\frac{q}{2 R^{3}}\left(K^{a} x_{a}\right)\left(Q^{b} v_{b}\right)=\frac{q\left(1-V^{2}\right) V \sin \theta}{2(1-V \cos \theta)^{3}} \frac{u}{r^{3}}+O\left(r^{-4}\right)
$$

so that the weak asymptotic stationarity condition (A.3) rules out burst memory. 
Homogeneous electromagnetic wave memory can be described using an antisymmetric Hertz potential $H^{a b}=H^{[a b]}$. The vector potential $A^{a}=\partial_{b} H^{a b}$ then satisfies the Lorentz gauge condition $\partial_{b} A^{b}=0$ and generates a solution of Maxwell's equations provided $H^{a b}$ satisfies the wave equation $\eta^{a b} \partial_{a} \partial_{b} H^{c d}=0$. Following [13], the purely E-mode Hertz potential

$$
\begin{aligned}
& H_{(E)}^{a b}:=\left(T^{a} Z^{b}-Z^{a} T^{b}\right) \frac{f(u)-f(u+2 r)}{r}, \\
& f(\tau)=\left\{\begin{array}{ccc}
C \tau & : & T<\tau \\
F(\tau) & : & 0 \leq \tau \leq T \\
0 & : & \tau<0,
\end{array}\right.
\end{aligned}
$$

where $F(\tau)$ is chosen to make the solution smooth, yields the non-zero E-mode memory

$$
\Delta \mathscr{V}_{[e]}=-C \cos \theta .
$$

The corresponding Newman-Penrose component satisfies

$\Phi_{[e] 0}=0, \quad u>T$, and $\quad \Phi_{[e] 0}=-\frac{1}{2} \frac{C u}{r^{3}} \sin \theta, \quad u<0, u+2 r>T$,

so that

$$
\Delta \Phi_{[e] 0}=\frac{1}{2} \frac{C u}{r^{3}} \sin \theta .
$$

Thus the weak asymptotic stationarity condition (A.3) rules out homogeneous wave E-mode memory.

Homogeneous B-mode solutions of the Maxwell equation are generated from the dual $H_{(B)}^{a b}={ }^{*} H_{(E)}^{a b}$ of $(\underline{\text { A.7 }})$. The resulting vector potential is purely magnetic with B-mode memory and B-mode Newman-Penrose component

$$
\Delta \mathscr{V}_{[b]}=i \Delta \mathscr{V}_{[e]} \quad \text { and } \quad \Phi_{[b] 0}=-i \Phi_{[e] 0} .
$$

Consequently the weak asymptotic stationarity condition (A.3) also rules out homogeneous wave B-mode memory.

We now show that the weak asymptotic stationarity condition rules out all memory sources except E-mode null memory. For that purpose, we consider Maxwell's equations with 4-current $J^{a}$, whose angular components are represented by a spin-weight 0 potential according to $\check{\partial} \mathscr{J}=q_{A} J^{A}$. In the null gauge, $A_{r}=0$, Maxwell equations decompose into the hypersurface, evolution and supplementary equations given by, respectively,

$$
\begin{aligned}
& 4 \pi r^{2} J^{u}=\partial_{r}\left(r^{2} \partial_{r} A_{u}-\bar{\partial} \partial \mathscr{A}_{[e]}\right) \\
& 4 \pi r^{4} \mathscr{J}=r^{2} \partial_{r}\left(2 \partial_{u} \mathscr{A}-A_{u}-\partial_{r} \mathscr{A}\right)+i \bar{\delta} \partial \mathscr{A}_{[b]} \\
& 4 \pi r^{2} J^{r}=\partial_{r}\left(\bar{\partial} \partial \mathscr{A}_{[e]}\right)-\partial_{u}\left(\bar{\partial} \partial \mathscr{A}_{[e]}\right)+\bar{\partial} \partial A_{u}-r^{2} \partial_{r} \partial_{u} A_{u} .
\end{aligned}
$$


As discussed in [13], if the current density is modeled after a charged fluid then the finiteness of its total angular momentum requires in Cartesian coordinates $x^{i} J^{k}-x^{k} J^{i}=O\left(r^{-3-\epsilon}\right), \epsilon>0$, which implies in null spherical coordinates that $J^{A}=O\left(r^{-5-\epsilon}\right)$. In addition, the finite flux of charge to $\mathcal{I}^{+}$requires $J^{r}=O\left(r^{-2}\right)$ with $\left.J_{[2]}^{r}\right|_{ \pm \infty}=0$. Thus the current satisfies the asymptotic conditions

$$
\Delta J_{[2]}^{r}=\Delta \mathscr{J}_{[4]}=0 \text {. }
$$

Global E-mode memory: As discussed in [13], the electromagnetic E-mode memory is governed by the supplementary equation (A.14) evaluated at $\mathcal{I}^{+}$,

$$
\left.\left\{4 \pi r^{2} J^{r}+\partial_{u} \precsim \bar{\partial} \mathscr{A}_{[e]}+r^{2} \partial_{u} \partial_{r} A_{u}\right\}\right|_{\mathcal{I}^{+}}=0 .
$$

Integration of (A.16) shows that the radiation memory (A.2) is governed by two terms,

$$
\overline{\mathrm{\partial}} \mathrm{\partial}\left(\Delta \mathscr{V}_{[e]}\right)=-\Delta A_{u[1]}+4 \pi \int_{-\infty}^{\infty} J_{[2]}^{r} d u,
$$

where the second term is the null memory and (A.17) is the electromagnetic counterpart to the E mode of the linearized gravitational wave memory (106).

The real part of the evolution equation (A.13) gives at $\mathcal{I}^{+}$

$$
4 \pi \mathscr{J}_{[e 4]}=-2 \partial_{u} \mathscr{A}_{[e 1]}+A_{u[1]} .
$$

Weak asymptotic stationarity (A.3) along with the current condition (A.15) imply that $\Delta A_{u[1]}=0$. Consequently, (A.17) shows that weak asymptotic stationarity eliminates all E-mode memory except null memory.

Global B-mode memory: The B-mode component of the electromagnetic memory (A.2),

$$
\Delta \mathscr{V}_{[b]}=-\Delta \mathscr{A}_{[b 0]}
$$

is governed by the imaginary part of the evolution equation (A.13),

$$
0=r^{2} \partial_{r}\left(4 \partial_{u} \mathscr{A}_{[b]}-\partial_{r} \mathscr{A}_{[b]}\right)-\overline{\mathrm{\partial}} \mathscr{\partial}_{[b]}-4 \pi r^{4} \mathscr{J}_{[b]} .
$$

Its evaluation at $\mathcal{I}^{+}$, along with the imaginary part of the weak asymptotic stationarity condition (A.3) and the current condition (A.15), yields

$$
0=\Delta \overline{\bar{\partial}} \check{\partial} \mathscr{A}_{[b 0]} \text {. }
$$

Thus weak asymptotic stationarity eliminates all B-mode electromagnetic radiation memory.

\section{Appendix B. Christoffel symbols}

Here we list useful Christoffel symbols for the calculation of the curvature quantities. For simplicity, we define $U_{A}=q_{A B} U^{B}$.

$$
\begin{aligned}
& \Gamma_{u u}^{u}=2 \partial_{u} \beta-\frac{1}{2} \partial_{r} W-\partial_{r} \beta \\
& \Gamma_{u r}^{u}=0
\end{aligned}
$$




$$
\begin{aligned}
& \Gamma_{u A}^{u}=D_{A} \beta-\frac{1}{2} \partial_{r}\left(r^{2} U_{A}\right) \\
& \Gamma_{r r}^{u}=0 \\
& \Gamma_{r A}^{u}=0 \\
& \Gamma_{A B}^{u}=r q_{A B}-2 r \beta q_{A B}+\frac{1}{2} \partial_{r}\left(r^{2} J_{A B}\right) \\
& \Gamma_{u u}^{r}=\frac{1}{2} \partial_{u} W-\partial_{u} \beta+\frac{1}{2} \partial_{r} W+\partial_{r} \beta \\
& \Gamma_{u r}^{r}=\frac{1}{2} \partial_{r} W+\partial_{r} \beta \\
& \Gamma_{u A}^{r}=\frac{1}{2} D_{A} W+\frac{1}{2} \partial_{r}\left(r^{2} U_{A}\right) \\
& \Gamma_{r r}^{r}=2 \partial_{r} \beta \\
& \Gamma_{r A}^{r}=\frac{1}{2} r^{2} \partial_{r} U_{A}+D_{A} \beta \\
& \Gamma_{A B}^{r}=-r q_{A B}-r(W-2 \beta) q_{A B}+r^{2} D_{(A} U_{B)}+\frac{1}{2} r^{2} \partial_{u} J_{A B} \\
& -\frac{1}{2} \partial_{r}\left(r^{2} J_{A B}\right) \\
& \Gamma_{u u}^{A}=-\partial_{u} U^{A}+\frac{1}{r^{2}} D^{A}\left(\beta+\frac{1}{2} W\right) \\
& \Gamma_{u r}^{A}=-\frac{1}{2 r^{2}} \partial_{r}\left(r^{2} U^{A}\right)+\frac{1}{r^{2}} D^{A} \beta \\
& \Gamma_{u B}^{A}=-\frac{1}{2} q^{A C}\left[D_{B} U_{C}-D_{C} U_{B}\right]+\frac{1}{2} q^{A C} \partial_{u} J_{B C} \\
& \Gamma_{r r}^{A}=0 \\
& \Gamma_{r B}^{A}=\frac{1}{r} \delta_{B}^{A}+\frac{1}{2} q^{A C} \partial_{r} J_{C B} \\
& \Gamma_{B C}^{A}={ }^{(q)} \Gamma_{B C}^{A}+r U^{A} q_{B C}+D_{(B} J_{C)}{ }^{A}-\frac{1}{2} D^{A} J_{B C}
\end{aligned}
$$

\section{References}

[1] Zeldovich ,Y and Polnarev A G Sov. Astron., Radiation of gravitational waves by a cluster of superdense stars, 18 (1974) 17

[2] L. Smarr, Gravitational radiation from distant encounters and from head-on collisions of black holes - The zero-frequency limit, Phys. Rev. D 152069 (1977)

[3] R. J. Bontz and R. H. Price, The spectrum of radiation at low frequencies, APJ 228 p. 560 (1979)

[4] Christodoulou, D., Phys. Rev. Lett., Nonlinear nature of gravitation and gravitational-wave experiments, 67, 1486 (1991)

[5] Thorne K S , Gravitational-wave bursts with memory: the Christodoulou effect Phys. Rev. D 45 $2(1992)$

[6] Wiseman A G and Will C M, Christodoulou's nonlinear gravitational-wave memory: evaluation in the quadrupole approximation Phys. Rev. D 44 R2945 (1991)

[7] Bieri L, Chen P and Yau S T, Null asymptotics of solutions of the EinsteinMaxwell equations in general relativity and gravitational radiation Adv. Theor. Math. Phys. 154 (2011)

[8] Bieri L and Garfinkle D 2015 Neutrino radiation showing a Christodoulou memory effect in general relativity Ann. Henri Poincaré 16801 
[9] Tolish A and Wald R M Retarded field of null particles and the memory effect Phys. Rev. D 89 064008 (2014)

[10] B. P. Abbott et al. "Observation of Gravitational Waves from a Binary Black Hole Merger", Phys. Rev. Lett. 116, 061102 (2016)

[11] Arzoumanian, Z. et .al.; NANOGrav Constraints on Gravitational Wave Bursts with Memory; APJ, 810, 150, (2015).

[12] M. Favata, The gravitational-wave memory effect, Class. Quant. Grav. 27, 084036 (2010)

[13] J. Winicour, Class. Quant. Grav.,Global aspects of radiation memory, 31, 205003 (2014)

[14] Sachs R, Asymptotic symmetries in gravitational theory Phys. Rev. 1282851 (1962)

[15] M. van der Burg, H. Bondi, and A. Metzner, Gravitational Waves in General Relativity. VII. Waves from Axi-Symmetric Isolated Systems, Proc. R. Soc. London Ser. A 269, 21 (1962).

[16] R. Sachs, Gravitational Waves in General Relativity. VIII. Waves in Asymptotically Flat SpaceTime, Proc. R. Soc. Ser. A 270, 103 (1962).

[17] Bieri L and Garfinkle D, Perturbative and gauge invariant treatment of gravitational wave memory Phys. Rev. D 89084039 (2014)

[18] Bieri L and Garfinkle D 2013 An electromagnetic analog of gravitational wave memory Class. Quantum Grav. 30195009

[19] Bieri, L; Garfinkle, D; Yau, S-T; Gravitational wave memory in de Sitter spacetime; eprint arXiv:1509.01296 (2015)

[20] Chu, Yi-Zen; Transverse-Traceless Gravitational Waves In A Spatially Flat FLRW Universe: Causal Structure from Dimension Reduction; Phys. Rev. D 92, 124038 (2015)

[21] Tolish, A and Wald, R. M.; The Cosmological Memory Effect; eprint arXiv:1606.04894 (2016)

[22] Kehagias A and Riotto A 2016 BMS in Cosmology J. Cosmol. Astropart. Phys., 05059

[23] Bieri L, Chen P and Yau S T, The electromagnetic Christodoulou memory effect and its application to neutron star binary mergers Class. Quantum Grav. 29 21, (2012)

[24] Chu, Yi-Zen; Gravitational Wave Memory In $\mathrm{dS}_{4+2 n}$ and 4D Cosmology; eprint arXiv:1603.00151 (2016)

[25] Pasterski, S; Strominger, A; Zhiboedov, A; New Gravitational Memories, eprint arXiv:1502.06120 (2015)

[26] Flanagan, E.; Nichols, D; Observer dependence of angular momentum in general relativity and its relationship to the gravitational-wave memory effect; Phys. Rev. D, 92, 084057 (2015)

[27] Strominger, Andrew; Zhiboedov, Alexander; Gravitational memory, BMS supertranslations and soft theorems; J. High Energy Phys., article id. \#86, 15 pp. (2016)

[28] S. W. Hawking, The Information Paradox for Black Holes, arXiv[gr-qc]:1509.01147, (2015)

[29] E. Newman and R. Penrose, Note on the Bondi-Metzner-Sachs Group, J. Math. Phys. 7, 863 (1966).

[30] R. Penrose, Asymptotic Properties of Fields and Space-Time, Phys. Rev. Lett. 10, 66 (1963).

[31] C. W. Misner, and K. S. Thorne, and J. A. Wheeler, Gravitation, San Francisco: W.H. Freeman and Co (1973)

[32] R. Gómez, L. Lehner, P. Papadopoulos, and J. Winicour, The eth formalism in numerical relativity, Class. Quant. Grav., 14977 (1997)

[33] J. Winicour, Newtonian gravity on the null cone, J. Math. Phys. 24, 1193 (1983).

[34] J. Winicour, Null infinity from a quasi-Newtonian view, J. Math. Phys. 25, 2506 (1984).

[35] L. A. Tamburino and J. Winicour, Gravitational Fields in Finite and Conformal Bondi Frames, Phys. Rev. 150, 1039 (1966).

[36] J. N. Goldberg, Conservation equations and equations of motion in the null formalism, Gen. Rel. Grav., 5, 183 (1974)

[37] R. Penrose and W. Rindler, Spinors and Space-Time (Cambridge University Press, Cambridge, 1984), Vol. 1.

[38] S. W. Hawking, and G.F.R. Ellis, The Large Scale Structure of Space-Time, Cambridge University Press 1973 
[39] E. Newman, and R. Penrose, An Approach to Gravitational Radiation by a Method of Spin Coefficients, J. Math. Phys, 3, 566 (1962)

[40] E. Newman, and R. Penrose, Errata: An Approach to Gravitational Radiation by a Method of Spin Coefficients, J. Math. Phys, 4, 998 (1963)

[41] J. Winicour, Massive fields at null infinity, J. Math. Phys, 27, 2117 (1988)

[42] Helfer, A. D.,Null infinity does not carry massive fields, J. Math. Phys., 34, 3478 , (1993)

[43] R. Sachs, P. G. Bergmann, Structure of Particles in Linearized Gravitational Theory, Phys. Rev., 112674 (1958)

[44] J. Boardman, P. G. Bergmann, Spherical Gravitational Waves, Phys. Rev. 115, 1318, (1959)

[45] Hawking S W, Perry M J and Strominger A 2016 Soft hair on black holes Phys. Rev. Lett. 116 231301 